\title{
Three-dimensional flow near a reconnection site at the dayside magnetopause: analytical solutions coupled with MHD simulations
}

\author{
LARS G. WESTERBER G ${ }^{1}$, J. VEDI N ${ }^{2}$, A. EK E N B Ä C K \\ and H. O. A K E R S T E D T ${ }^{1}$ \\ ${ }^{1}$ Division of Fluid Mechanics, Luleå University of Technology, SE-971 87, Luleå, Sweden \\ ${ }^{2}$ Department of Physics, Umeå University, SE-901 87, Umeå, Sweden \\ ${ }^{3}$ Swedish Institute of Space Physics, SE-981 28, Kiruna, Sweden
}

(Received 25 October 2006, accepted 20 November 2006)

\begin{abstract}
We present a coupling between an analytical three-dimensional model covering the plasma flow behaviour through the magnetopause transition layer near a reconnection site, with results from a global MHD simulation describing the plasma flow in the magnetosheath. The structure of the plasma flow near a reconnection site at the dayside terrestrial magnetopause is investigated, together with the development of the magnetopause transition region.
\end{abstract}

\section{Introduction}

In a previous paper, Westerberg and Åkerstedt (2006a) presented an analytical solution for the Solar wind plasma flow in the vicinity of a reconnection site located at the dayside magnetopause. The equations governing the flow structure in the transition layer separating the plasma in the magnetosphere and the magnetosheath were matched with an analytical model for the flow at the outer magnetopause boundary. The main objective with the present study is to combine the analytical solutions valid in the transition layer with results from an MHD simulation, covering the flow in the magnetosheath. Magnetic reconnection is assumed to occur in a region stretching from the sub-Solar point towards the north. Its effect on the surrounding plasma flow is investigated. In Westerberg and Åkerstedt (2006a) the outer solutions represent the case where the Solar wind plasma velocity and magnetic field are aligned. In this study we focus on the case where the Solar wind velocity and magnetic field are perpendicular. We obtain the structure of the plasma velocity and magnetic field during the transition of the magnetopause, together with the development of the transition region when moving away from the reconnection site.

The dimensionless equations describing the plasma properties north and south of the reconnection line are Westerberg and Åkerstedt (2006a)

$$
\begin{aligned}
\hat{\mathbf{y}}: & \alpha \frac{\partial^{2} u_{y}^{(0)}}{\partial \xi^{2}}+2 \xi\left(\frac{\partial U_{y}}{\partial y}+\frac{\partial U_{z}}{\partial z}\right) \frac{\partial u_{y}^{(0)}}{\partial \xi}+u_{y}^{(0)} \frac{\partial U_{z}}{\partial z}-2 U_{y} \frac{\partial u_{y}^{(0)}}{\partial y} \\
& -U_{y} \frac{\partial u_{z}^{(0)}}{\partial z}-U_{y} \frac{\partial U_{y}}{\partial y}-u_{z}^{(0)} \frac{\partial U_{y}}{\partial z}-U_{z}\left(\frac{\partial u_{y}^{(0)}}{\partial z}+\frac{\partial U_{y}}{\partial z}\right)-\frac{\partial P}{\partial y}=0
\end{aligned}
$$




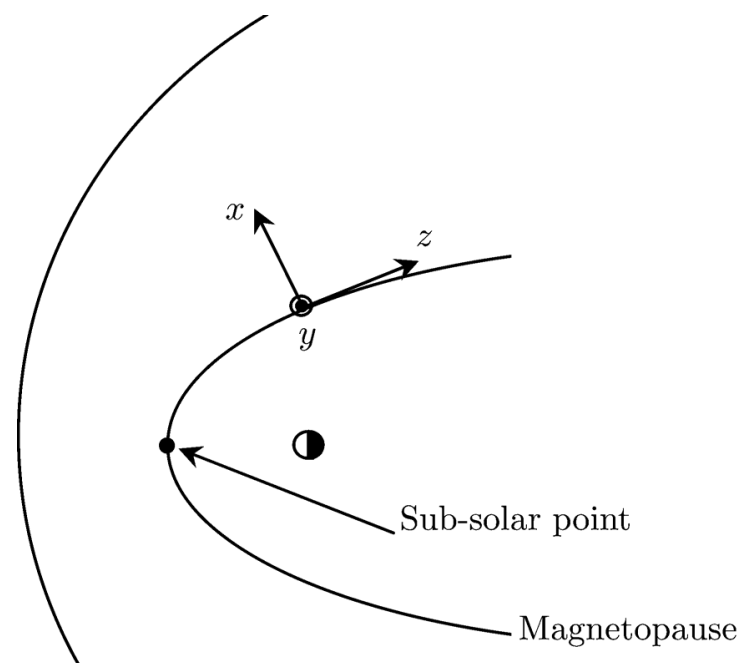

Magnetosheath

Figure 1. The curvilinear coordinate system with conventional labels for the coordinate axis, with $x$ pointing in a direction normal to the magnetopause surface, $z$ in a tangential direction, and $y$ completing the right-hand system.

$$
\begin{aligned}
\hat{\mathbf{z}}: \quad & \alpha \frac{\partial^{2} u_{z}^{(0)}}{\partial \xi^{2}}+2 \xi\left(\frac{\partial U_{y}}{\partial y}+\frac{\partial U_{z}}{\partial z}\right) \frac{\partial u_{z}^{(0)}}{\partial \xi}+u_{z}^{(0)} \frac{\partial U_{y}}{\partial y}-2 U_{z} \frac{\partial u_{z}^{(0)}}{\partial z} \\
& -U_{z} \frac{\partial u_{y}^{(0)}}{\partial y}-U_{y}\left(\frac{\partial u_{z}^{(0)}}{\partial y}+\frac{\partial U_{z}}{\partial y}\right)-U_{z} \frac{\partial U_{z}}{\partial z}-u_{y}^{(0)} \frac{\partial U_{z}}{\partial y}-\frac{\partial P}{\partial z}=0 .
\end{aligned}
$$

$x, y$ and $z$ are the curvilinear coordinates along the magnetopause boundary (Fig. 1), with $x$ pointing in a direction normal to the magnetopause surface, $z$ in a tangential direction, and $y$ completing the right-hand system. The independent variables $\xi$, $y$, and $z$ are dimensionless variables defined in units of the Earth radii $\left(R_{\mathrm{E}}\right)$. In Westerberg and Åkerstedt (2006a) these equations were derived for small reconnection rates. It can be shown, however, that the equations are also valid for larger reconnection rates. In (1.1) and (1.2), $P$ is the total pressure $\left(p+B^{2} /\left(2 \mu_{0}\right)\right) . d P / d y$ and $d P / d z$ are obtained from the inviscid MHD equations of motion (Westerberg and Åkerstedt 2006a). $U_{y, z}$ represent components of the DeHoffmann-Teller (DHT) velocity (DeHoffmann and Teller 1950; Westerberg and Åkerstedt 2006a), $\alpha=$ $\left(1+\eta_{\mathrm{d}} / \nu\right)$ where $\eta_{\mathrm{d}}$ is the magnetic diffusivity and $\nu$ the kinematic viscosity, and $u_{y, z}^{(0)}$ represent components of the plasma velocity with respect to the DHT frame of reference. Using $\nu \sim \eta_{\mathrm{d}}$ as motivated by Haerendel et al. (1978) we obtain $\alpha=2$. $\xi$ is the re-scaled $x$-coordinate such that $\xi=R^{1 / 2} x$, where $R$ is the Reynolds number. See Westerberg and Åkerstedt (2006a) for details in the analysis. 


\section{Solutions}

For the solutions to (1.1) and (1.2) we utilize the Comsol Multiphysics Software $†$ to solve the equations numerically. The DHT velocities are obtained from the relations

$$
\begin{aligned}
& \mathbf{U}^{*}=\mathbf{U}_{\mathrm{HT}}^{\mathrm{N}}+\mathbf{B}^{*}, \\
& \mathbf{U}^{*}=\mathbf{U}_{\mathrm{HT}}^{\mathrm{S}}-\mathbf{B}^{*},
\end{aligned}
$$

where the asterisk refers to the outer magnetopause boundary variables. $\mathrm{N}$ and $\mathrm{S}$ correspond to positions north and south of the reconnection line, respectively. See Section 2.1 for the variation of $\mathbf{U}^{*}$ and $\mathbf{B}^{*}$ along the magnetopause, obtained from the MHD simulation. We consider a geometry where $-4 \leqslant x \leqslant 4,-3 \leqslant y \leqslant 3$, and $-3 \leqslant z \leqslant 3$. As initial conditions at the reconnection line $(z=0)$, the variables are assumed to be independent of $y$, from which it follows that there is no $y$-dependence in the solutions. Homogeneous Neumann conditions are used at all boundaries except at $z=0$ where Dirichlet conditions are utilized such that $u_{y, z}^{(0)}=\operatorname{erf}(\beta x)$ in accordance with the lowest-order analytical solutions. In order to represent a sharp transition at $z=0$, but still smooth enough to be treated numerically without refining the mesh too excessively, we consider $\beta=5$.

\subsection{Ideal MHD simulations}

To obtain the variation of $\mathbf{U}^{*}$ and $\mathbf{B}^{*}$ along the magnetopause we use an ideal MHD simulation based on the FLASH Software $\neq$. We simulate a 3D box where each side is of length $L=2 \times 10^{8} \mathrm{~m}$. At the boundary $X=-L$ we apply a Solar wind flow velocity $u_{X}=450 \mathrm{~km} \mathrm{~s}^{-1}$, with a density of 7 protons $\mathrm{cm}^{-3}$, a temperature of $1.5 \times 10^{5} \mathrm{~K}$ and a magnetic field $B_{Z}=-5 \mathrm{nT}$. The shape of the magnetopause is modelled by the equation $X=\left(Y^{2}+Z^{2}\right) /\left(20 R_{\mathrm{E}}\right)$. We consider the limit of a very small reconnection rate, which means that, in the lowest-order approximation, we assume that the normal components of the velocity and the magnetic field are zero at the magnetopause boundary. In Fig. 2 we present results from the $X Z$-plane of the MHD simulations showing the $Z$-component of the magnetic field together with a vector plot of the velocity.

When the bow shock has been generated and stabilized we extract the data containing the plasma velocity and magnetic field along the magnetopause boundary in the $X Z$-plane. We use the method of least squares to fit this data to linear polynomials and obtain

$$
\begin{aligned}
& U_{z}^{*}=0.0814+0.0344 s \\
& U_{y}^{*}=0.0290-0.0025 s \\
& B_{z}^{*}=-5.3959+0.2130 s \\
& B_{y}^{*}=0.0983-0.0038 s
\end{aligned}
$$

where $s$ is the distance along the magnetopause from the sub-Solar point. In these expressions, the velocities have been normalized with $\left|u_{X}\right|$ and the magnetic fields with $\left|B_{Z}\right|$.

$\dagger$ Comsol Multiphysics ${ }^{\text {TM }}$ 3.2, provided by Comsol AB, Stockholm, Sweden $\ddagger$ ASC/Alliances Center for Astrophysical Thermonuclear Flashes, http://flash.uchicago.edu/website/home. 

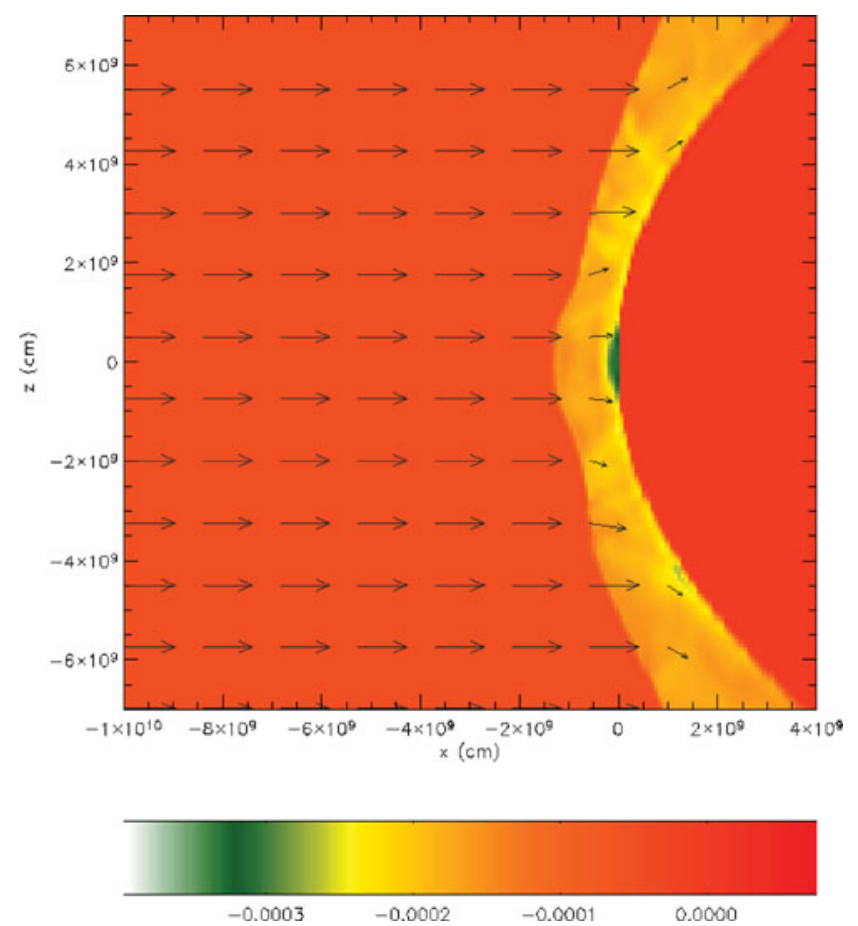

Figure 2. The $X Z$-plane in the MHD simulations showing the $Z$-component of the magnetic field (in Gauss) as shading and the velocity as arrows.

\subsection{Alfvén Mach number along the magnetopause}

In order for stationary reconnection to occur, the Alfvén Mach number $\left(M_{\mathrm{A}}\right)$ has to be smaller than unity (Westerberg and Åkerstedt 2005). A question that has been raised by several authors is whether reconnection can occur downstream at the polar cusp for northward interplanetary magnetic field; for example Cooling et al. (2001) showed that $M_{\mathrm{A}}<1$ southward of the cusp. Their model is, however, built on other models governing the magnetosheath flow. It is thus of certain interest to investigate the development of the Alfvén Mach number $\left(M_{\mathrm{A}}=\left|\mathbf{U}^{*}\right| /\left[\left|\mathbf{B}^{*}\right| / \sqrt{\rho}\right]\right)$ along the magnetopause boundary. Figure 3 shows $M_{\mathrm{A}}$ for the case when $\mathbf{u}_{\mathrm{sw}} \| \mathbf{B}_{\mathrm{sw}}$ (Fig. 3(a)) and $\mathbf{u}_{\mathrm{sw}} \perp \mathbf{B}_{\mathrm{sw}}$ (Fig. 3(b)), where $\mathbf{u}_{\mathrm{sw}}$ and $\mathbf{B}_{\mathrm{sw}}$ is the Solar wind velocity and magnetic field, respectively. The polar cusp is located at $s \approx 10 R_{\mathrm{E}}$. We see that the Alfvén Mach number never reaches unity in any of the two cases. Therefore, magnetic reconnection can occur for all positions along the magnetopause, even in the region downstream of the cusp for the case of a northward interplanetary magnetic field. In our treatment we have assumed that the reconnection rate is small and that the reconnection process has only a small influence on the outer magnetosheath flow. At least for small reconnection rates the gradients of the outer flow are of the order of the square of the reconnection rate (Westerberg and Åkerstedt 2006b). From the results of the simulations the velocity gradient of the Alfvén Mach number is very small for large $s$ for the case of aligned fields, while it is small for small $s$ for the case of perpendicular fields. In these regions the effect of reconnection on the magnetosheath flow should be small, while in the outer regions the effects of reconnection become more important. 

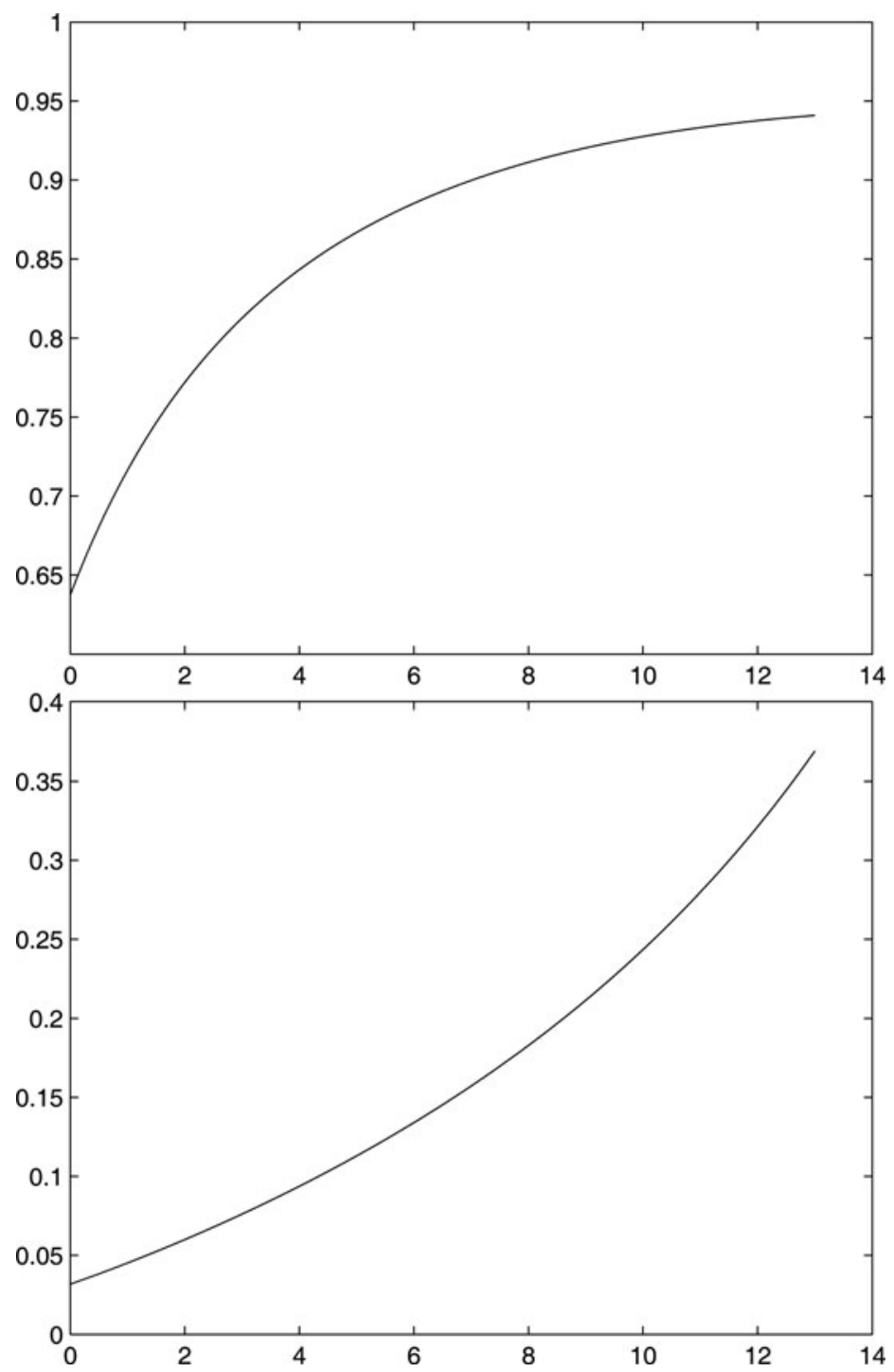

Figure 3. Alfvén Mach number $\left(M_{\mathrm{A}}=\left|\mathbf{U}^{*}\right| /\left[\left|\mathbf{B}^{*}\right| / \sqrt{\rho}\right]\right)$ along the magnetopause as a function of the distance $s$ (in Earth radii) from the sub-Solar point. (a) Parallel Solar wind velocity and magnetic field: $\mathbf{u}_{\mathrm{sw}} \| \mathbf{B}_{\mathrm{sw}}$. (b) Perpendicular Solar wind velocity and magnetic field: $\mathbf{u}_{\mathrm{sw}} \perp \mathbf{B}_{\mathrm{sw}}$. The polar cusp is located at $s \approx 10 R_{\mathrm{E}}$. Magnetic reconnection should thus not be limited to occur in a region south of the polar cusp. In the present analysis we consider two locations of the reconnection site, $s=2 R_{\mathrm{E}}$ and $s=12 R_{\mathrm{E}}$.

For the solutions we treat two locations of the reconnection site, $s=2 R_{\mathrm{E}}$ and $s=12 R_{\mathrm{E}}$, i.e. the upper value of $s$ being past the polar cusp. As in Westerberg and Akerstedt (2006a) the reconnection line is considered to be aligned with the $y$-axis.

\subsection{Reconnection site at $2 R_{\mathrm{E}}$ from the sub-Solar point}

Solving (1.1) and (1.2) results in the development of the total velocity $\left(\mathbf{u}^{*}=\mathbf{u}+\mathbf{U}_{\mathrm{HT}}\right)$ and magnetic field during the transition of the magnetopause (see Fig. 4). The 


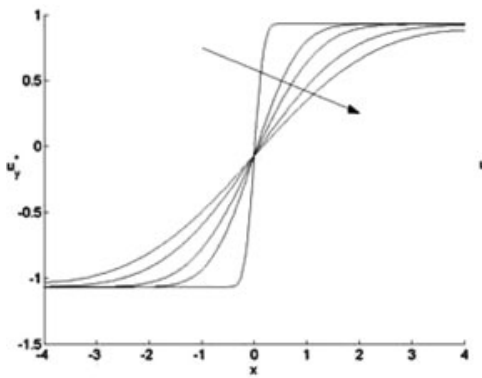

(a)

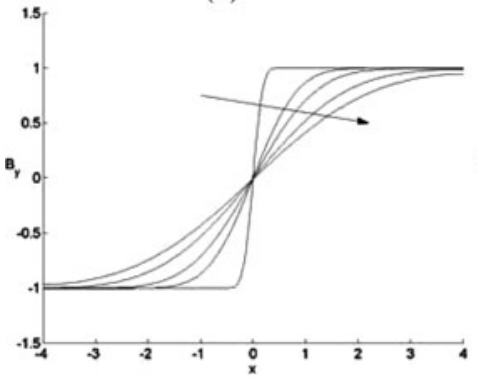

(c)

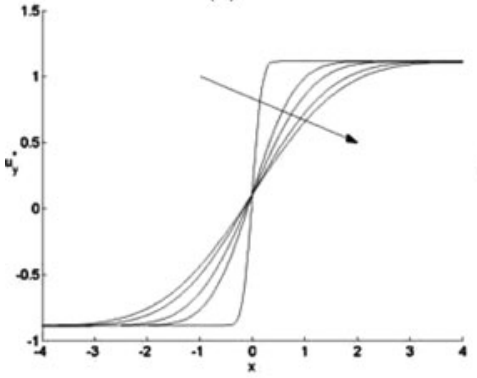

(e)

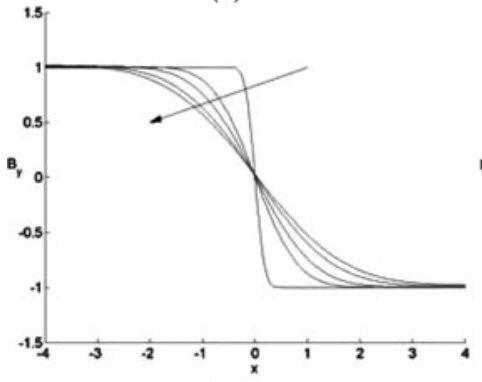

(g)

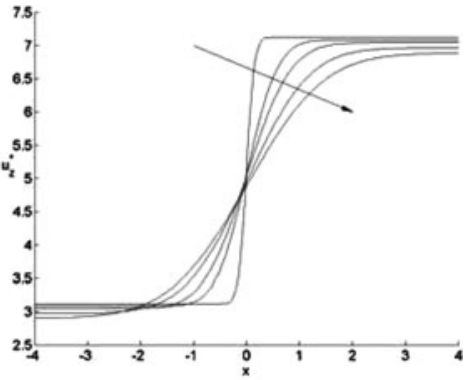

(b)

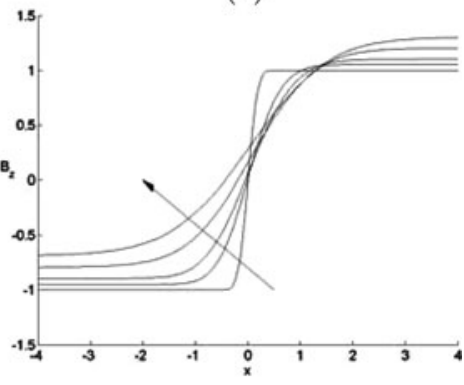

(d)

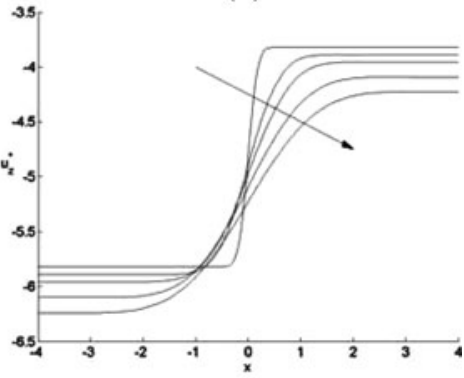

(f)

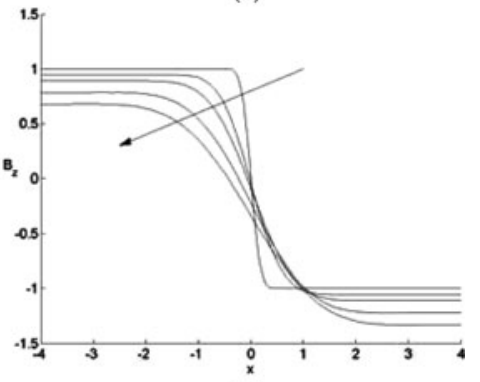

(h)

Figure 4. Development of total velocity $\left(\mathbf{u}^{*}=\mathbf{u}+\mathbf{U}_{\mathrm{HT}}\right)$ and magnetic field during the transition of the magnetopause. Reconnection site at a distance of $2 R_{\mathrm{E}}$ from the sub-Solar point. The arrow points in the direction of increasing $z$ (in $R_{\mathrm{E}}$ ) north of the reconnection site $(z=0, z=0.5, z=1, z=2, z=3)$, and decreasing $z$ south of the reconnection site $(z=0$, $z=-0.5, z=-1, z=-2, z=-3$ ). (a) North $u_{y}^{*}$; (b) north $u_{z}^{*}$; (c) north $B_{y}$; (d) north $B_{z}$; (e) south $u_{y}^{*} ;$ (f) south $u_{z}^{*} ;(\mathrm{g})$ south $B_{y} ;(\mathrm{h})$ south $B_{z}$. 
reconnection site is located at a distance of $2 R_{\mathrm{E}}$ from the sub-Solar point. The arrow points in the direction of increasing $z$ (in $R_{\mathrm{E}}$ ) north of the reconnection site $(z=0, z=0.5, z=1, z=2, z=3)$, and decreasing $z$ south of the reconnection site $(z=0, z=-0.5, z=-1, z=-2, z=-3)$.

\subsection{Reconnection site at $12 R_{\mathrm{E}}$ from the sub-Solar point}

The corresponding solutions for the case of a reconnection site at a distance of $12 R_{\mathrm{E}}$ from the sub-Solar point are shown in Fig. 5. As for the previous case, the arrow points in the direction of increasing $z$ (in $R_{\mathrm{E}}$ ) north of the reconnection site $(z=0$, $z=0.5, z=1, z=2, z=3)$, and decreasing $z$ south of the reconnection site $(z=0$, $z=-0.5, z=-1, z=-2, z=-3)$.

Observing the development of the velocity and magnetic field for the case of $s=2$ and $s=12$, we note that the thickness of the magnetopause transition region grows faster for the latter case. The thickness also increases when moving away from the reconnection site. From the analytical solution in Westerberg and Åkerstedt (2006a) we see that the transition layer thickness $(\delta)$ grows as $\sqrt{\left|z / U_{z}^{0}\right|}$, where $U_{z}^{0}$ is the DHT velocity at the reconnection site. For the present situation where $\mathbf{u}_{\mathrm{sw}} \perp \mathbf{B}_{\mathrm{sw}}, U_{z}^{0}$ is smaller when $s=12$ compared to $s=2$. However, when $\mathbf{u}_{\mathrm{sw}} \| \mathbf{B}_{\mathrm{sw}}$ the situation is the other way around - the thickness grows faster more close to the sub-Solar point since $U_{z}^{0}$ increases with increasing $s$.

\section{Summary}

In this paper we present an analysis combining the previously developed 3D resistive-viscous model describing the transition from the magnetosheath plasma to the boundary layer plasma in the vicinity of a reconnection site, with results from an ideal MHD simulation representing the flow in the magnetosheath plasma. We treat the case where the Solar wind velocity and magnetic field are perpendicular to each other. The main objective is to study the development of the plasma velocity and magnetic field during the transition of the magnetopause. Significant but not very dramatic differences in the behaviour of the plasma velocity and magnetic field development are observed for the two locations of the reconnection site. Observing the structure of the Alfvén Mach number along the magnetopause, we note that reconnection is possible after the cusp, both for the case of an aligned Solar wind velocity and magnetic field, and for the case of perpendicular fields. It is shown that the plasma flow experiences a more diffusive behaviour when the plasma velocity is higher and the magnetic field is stronger. Furthermore, for this case the transition region, which is initially very thin, grows faster than when the reconnection site is closer to the sub-Solar point.

There are several ways to go in order to develop the present model: introducing a dipole magnetic field from the Earth and thus allowing the magnetopause to form naturally when the Solar wind hits the dipole field, the structure of the magnetic field and plasma velocity along the inner magnetopause boundary are revealed from the simulation. A further development of the theoretical analysis is to find corresponding outer solutions with finite values of the normal components of the magnetic field and velocity field on the surface of the magnetopause. Also, the theoretical analysis can contain viscosity and resistivity as non-ideal effects. Since the plasma is mainly collisionless, inclusion of other effects such as the Hall terms in 


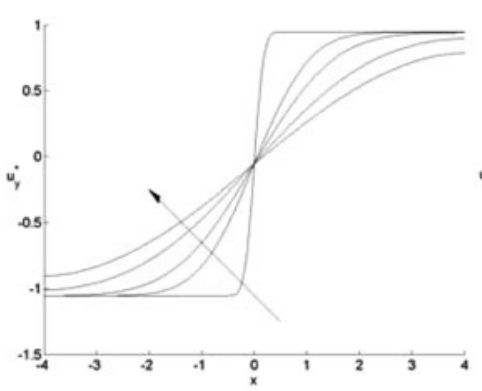

(a)

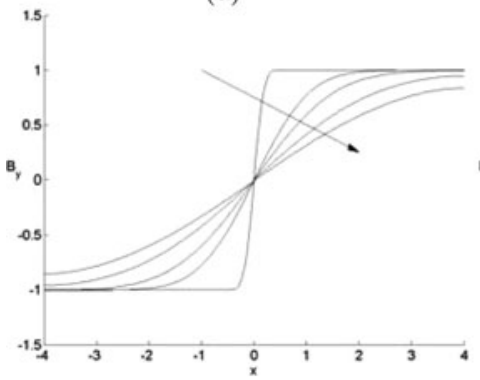

(c)

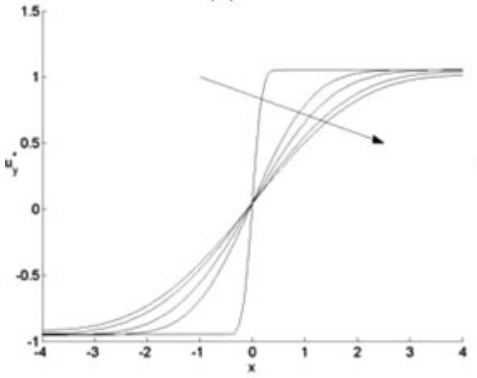

(e)

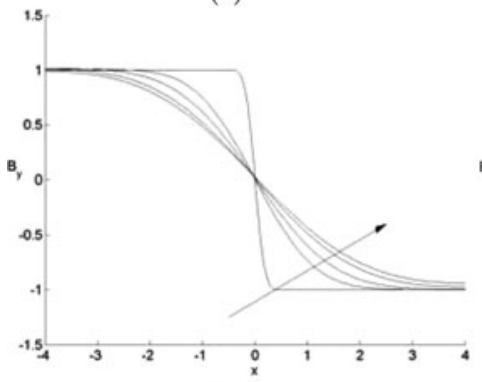

(g)

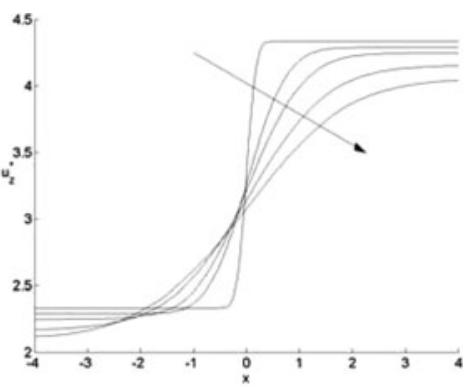

(b)

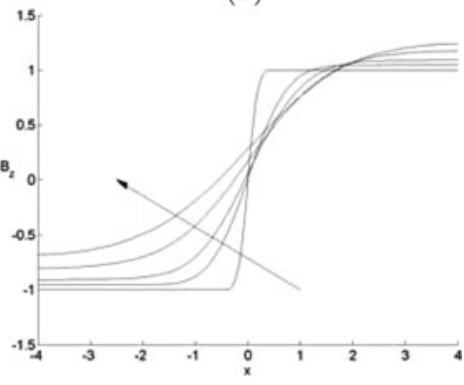

(d)

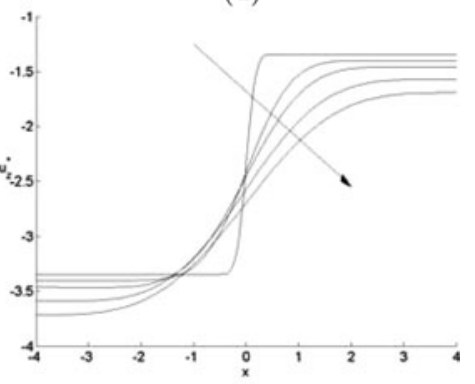

(f)

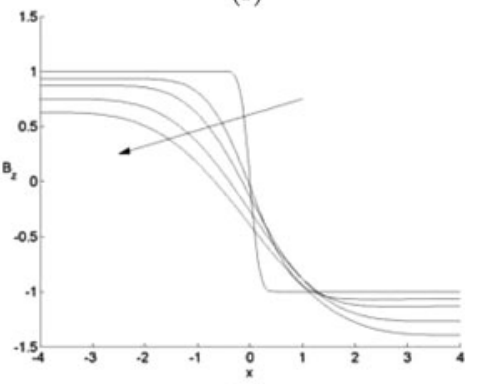

(h)

Figure 5. Development of total velocity $\left(\mathbf{u}+\mathbf{U}_{\mathrm{HT}}\right)$ and magnetic field during the transition of the magnetopause. Reconnection site at a distance of $12 R_{\mathrm{E}}$ from the sub-Solar point. The arrow points in the direction of increasing $z$ (in $R_{\mathrm{E}}$ ) north of the reconnection site $(z=0$, $z=0.5, z=1, z=2, z=3)$, and decreasing $z$ south of the reconnection site $(z=0$, $z=-0.5, z=-1, z=-2, z=-3$ ). (a) North $u_{y}^{*}$; (b) north $u_{z}^{*}$; (c) north $B_{y}$; (d) north $B_{z}$; (e) south $u_{y}^{*} ;$ (f) south $u_{z}^{*}$; (g) south $B_{y} ;$ (h) south $B_{z}$. 
the generalized Ohm's law are of certain interest. Finally, it is important to clarify how the magnetosheath flow is modified by the reconnection process.

\section{Acknowledgements}

The present research project is financed through the Swedish National Graduate School of Space Technology. The MHD simulations were conducted using the resources of the High Performance Computing Center North (HPC2N) and the software was in part developed by the DOE-supported ASC/Alliance Center for Astrophysical Thermonuclear Flashes at the University of Chicago.

\section{References}

Cooling, B. M. A., Owen, C. J. and Schwarz, S. J. 2001 Role of the magnetosheath flow in determining the motion of open flux tubes. J. Geophys. Res. 106(A9), 18.763-18.775.

DeHoffmann, F. and Teller, E. 1950 Magnetohydrodynamic shocks. Phys. Rev. Lett. 80, 692703.

Haerendel, G., Paschmann, N., Sckoke, N. and Rosenbauer, H. 1978 The front side boundary layer of the magnetosphere and the problem of reconnection. J. Geophys. Res. 82, 31953216.

Westerberg, L. G. and Åkerstedt, H. O. 2005 A two-dimensional analysis of the flow past an open terrestrial magnetopause. J. Plasma Physics 71(5), 537-562.

Westerberg, L. G. and Åkerstedt, H. O. 2006a A three-dimensional analysis of the flow past an open terrestrial magnetopause. J. Plasma Physics 72(3), 359-382.

Westerberg, L. G. and Åkerstedt, H. O. 2006b Two-dimensional effects of reconnection on the magnetosheath flow. In preparation. 